\title{
Ciclo de mejora docente para la asignatura de Relaciones Internacionales basado en la resolución de problemas y el aprendizaje crítico de los alumnos
}

\author{
LAURA GARCía MARTÍN \\ Derecho Internacional Público y \\ Relaciones Internacionales \\ Universidad de Sevilla \\ lgarcia17@us.es \\ ORCID: https://orcid.org/ 0000-0003-0163-9108 \\ D.O.I.: http://dx.doi.org/10.12795/JDU.2018.i01.61 \\ Pp.: 1086-1099
}

\section{Breve resumen}

Esta comunicación aborda el desarrollo de un ciclo de mejora docente en la asignatura de Relaciones Internacionales del Grado en Periodismo durante el primer cuatrimestre del curso 2018/2019. Dicho ciclo de mejora se basa en la definición de un modelo metodológico concreto en función de los contenidos fundamentales que se pretenden impartir, la planificación y descripción de las distintas actividades a realizar, así como los resultados conseguidos con el desarrollo de este nuevo sistema de aprendizaje. Esta forma de enseñanza implica que los alumnos mantengan una actitud proactiva y se impliquen en las clases mediante su asistencia y la participación en las actividades 
(talleres conceptuales, debates, etc.) de manera que ellos mismos vayan generando el conocimiento guiados por el docente para alcanzar los objetivos de aprendizaje. La presente comunicación trata de condensar los rasgos principales de la planificación, aplicación y valoración final del ciclo de mejora docente anteriormente propuesto.

Palabras clave: Relaciones Internacionales, Grado en Periodismo, docencia universitaria, experimentación docente universitaria.

\section{Breve descripción del contexto}

La asignatura Relaciones Internacionales se imparte en el primer cuatrimestre del primer curso del Grado en Periodismo. El grupo en el que la autora imparte docencia consta de 90 alumnos matriculados, de los cuales 70 asisten a clase con regularidad. Las clases se imparten los lunes y los jueves de 17:30 a 19:30, siendo la última hora de clases en la Facultad de Comunicación. Se trata de una asignatura particular porque los principios fundamentales de la materia pertenecen al campo de la ciencia política pero se imparte en un grado en periodismo y por iuspublicistas. En la misma se estudian los principales actores de las RRII, así como las dinámicas que influyen en las relaciones entre ellos.

\section{Diseño previo del Ciclo de Mejora Docente}

\section{Mapa de Contenidos}

Este ciclo de mejora se ha diseñado para la lección 4, El Estado, del módulo II, Los actores de las RRII. Los contenidos más relevantes de este tema se han organizado en un mapa de contenido que se muestra en la Figura 1:

Jornadas de Formación e Innovación Docente del Profesorado I № 1 (2018) Esta obra se distribuye con la licencia Creative Commons 


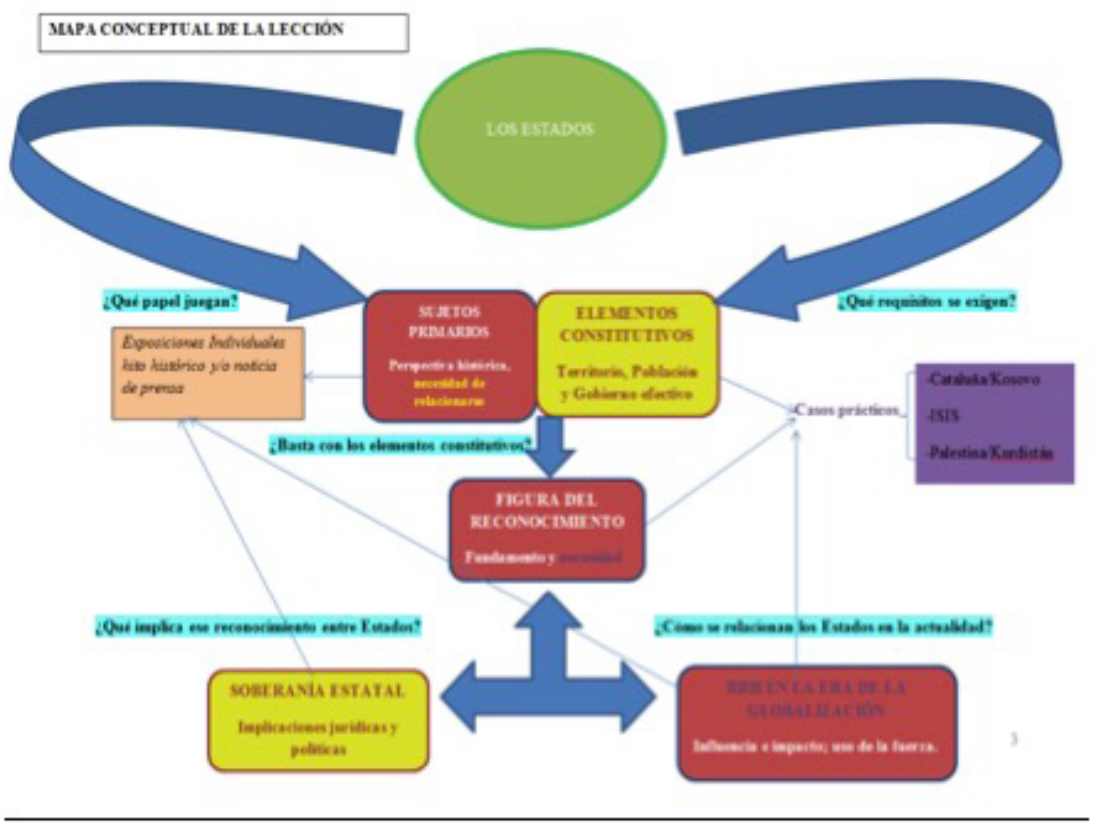

Figura 1. Mapa de contenido para la Lección 4: El estado.

Este mapa de contenidos refleja en esencia cuáles son los contenidos conceptuales y procedimentales, así como los problemas o cuestiones (en azul) y casos prácticos (en morado) que pueden guiar el aprendizaje y las conexiones y orden entre ellos, reflejada a través de las flechas. Por otro lado, los principales contenidos actitudinales, son los que tienen que ver con el espíritu crítico, la argumentación respecto a los problemas y las capacidades de expresión escrita y oral.

\section{Modelo Metodológico}

El modelo metodológico planteado para este sistema de docencia se resume en el esquema mostrado en la Figura 2:

Jornadas de Formación e Innovación Docente del Profesorado I № 1 (2018)
Esta obra se distribuye con la licencia Creative Commons 
L/R: Introducción y repaso

LA/HA: ideas o hipótesis de los alumnos

AC: actividad de contraste

AE: actividad de evaluación

AI: actividad exposición individual

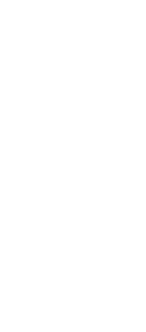

\section{I/R}

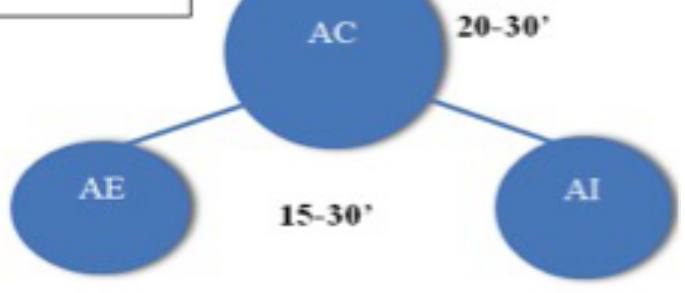

Figura 2. Modelo metodológico aplicado.

De esta manera, durante el desarrollo de las clases se alternan actividades en las que los conceptos puramente teóricos son introducidos a través de la práctica, bien a través de un taller conceptual (Don Finkel, 2008) o a través de debates de actualidad y visionado de medios audiovisuales. En cualquier caso, el profesor va guiando a los alumnos a través de explicaciones generales o preguntas abiertas para que sean los alumnos los que vayan construyendo el aprendizaje a través del diálogo interno (es decir, con ellos mismos, argumentando sus posturas) y externo (exponiéndolas al resto de la clase).

\section{Fichas de actividades}

El 2o ciclo de mejora abarca 3 sesiones de 2 horas de duración cada una.

Para cada actividad de este 20 ciclo de mejora hemos realizado una ficha detallada sobre la fase metodológica a la que pertenece, el tiempo invertido en la actividad, un resumen del contenido de la misma y el material utilizado. 
Primera sesión

\begin{tabular}{|l|l|l|}
\hline 1 & Fase metodológica: Introducción & $5-7^{\prime}$ \\
\hline $\begin{array}{l}\text { Descripción: comienzo la sesión haciendo una breve introducción al principio } \\
\text { de la misma en la que explico el contenido general de la lección y lo que va- } \\
\text { mos a hacer en esta primera clase. }\end{array}$ \\
\hline Recursos: voz
\end{tabular}

2

Fase metodológica: Ideas/ hipótesis alumnos 40

Descripción: Trabajo en grupo o 'taller conceptual'. Divido la clase en unos 8 grupos (cada uno formado por entre 5-10 personas en función de los asistentes) y les planteo el siguiente caso práctico.

'Tras la declaración unilateral de independencia de Cataluña, el Presidente del Consejo Europeo, Donald Tusk, convoca una reunión de urgencia para tratar este tema en el seno del Consejo'. Cada grupo representará los intereses de uno de los siguientes Estados: España, Francia, Italia, Alemania, Portugal, Bélgica, Reino Unido. Asimismo, uno de los grupos actuará en representación de la delegación del Gobierno Catalán para defender su causa en el Consejo.

Cada grupo elegirá uno o dos portavoces y tendrán que preparar sus alegaciones para presentar frente al resto de Estados. Deben en primer lugar, hacer un breve trabajo de investigación para saber cuál es la posición de su Estado con respecto al conflicto y al ppio de libre determinación de los pueblos de manera general. Como guía, deberán redactar entre 3 y 5 argumentos para reclamar su posición frente a este caso en el seno del Consejo.

Recursos: internet, ordenadores, tablets.

3

Fase metodológica: Actividad de contraste

30

Descripción: Cada portavoz del grupo presenta sus alegatos como si estuviésemos en el seno de la UE. Una vez que todos han presentado, debatimos y ponemos en común las intervenciones apoyándonos en conceptos teóricos clave de la asignatura que iré revelando con la ayuda del ppt, de manera que vayamos combinando las intervenciones prácticas con la teoría de la lección.

Recursos: ppt, pizarra.

\section{4}

Fase metodológica: Actividad individual de exposición

Descripción: los dos alumnos a los que les corresponde ese día realizan su exposición breve de una noticia que hayan seleccionado previamente. Esta actividad les ayuda a trabajar de forma individual y progresiva la asignatura, ya que deben hacer un breve comentario de la noticia y localizarla en el programa para que vayan relacionando los aspectos teóricos del mismo con la práctica de la vida real que aparece reflejada en la prensa. Además, les ayuda a ir perdiendo el miedo escénico de hablar en público. Para el comentario de noticias tienen unos 5-7 minutos por persona.

Jornadas de Formación e Innovación Docente del Profesorado I № 1 (2018)

Esta obra se distribuye con la licencia Creative Commons

Reconocimiento-NoComercial-SinObraDerivada

4.0

Internacional (CC BY-NC-ND 4.0.) 
Recursos: proyector, internet.

\section{Segunda sesión}

\begin{tabular}{|l|l|l|}
\hline 5 & Fase metodológica: Introducción/repaso & $5-7$ \\
\hline
\end{tabular}

Descripción: comienzo la sesión haciendo un breve repaso de lo que vimos en la clase anterior para refrescar la memoria y les planteo lo que vamos a hacer en esa clase para que sean capaces de seguir el orden lógico de la misma.

Recursos: $\mathrm{VOZ}$

6 Fase metodológica: Ideas/hipótesis de los $30^{-}$ alumnos

Descripción: Les pongo dos videos breves para introducir el contexto de algunos de los casos que vamos a tratar en la clase (principalmente Kosovo, Cataluña y Kurdistán). Tras el visionado, pido que algunos voluntarios comenten los conflictos y lo relacionen con los apartados de la lección que tienen relevancia.

Recursos: youtube, internet, proyector.

\begin{tabular}{|l|l|l|}
\hline 7 & Fase metodológica: Actividad de contraste & $30^{-}$ \\
\hline Descripción: A partir de la tarea anterior, comienzo a hacer preguntas generales \\
sobre la lección que estamos viendo, concretamente relacionado con el apar- \\
tado del reconocimiento y la soberanía estatal. Estas preguntas tienen que ver \\
con los casos prácticos incluidos en el mapa conceptual y mi intención es que \\
sean capaces de razonar ellos mismos sobre la problemática que presentan es- \\
tos casos reales y establezcan las similitudes y diferencias entre ellos.
\end{tabular}

Recursos: pizarra, ppt.

8

Fase metodológica: Exposición individual personaje o hito histórico de las RRII
25

Descripción: los dos alumnos a los que les corresponde ese día realizan su exposición breve sobre un personaje relevante o hito histórico de las RRII. Para la presentación tienen unos 10-12 minutos por persona. La idea es que sea conciso y les recomiendo que en el ppt usen más imágenes que texto, ya que creo que resulta más interesante y visual para el resto de compañeros.

Recursos: ppt, youtube, proyector, imágenes.

\section{Tercera sesión}

\begin{tabular}{|l|l|l|}
\hline 9 & Fase metodológica: Introducción/repaso & $5-7^{-}$ \\
\hline Descripción: comienzo siempre la sesión haciendo un breve repaso de lo que vi- \\
mos en la clase anterior para refrescar la memoria y les planteo lo que vamos a \\
hacer en esa clase para que sean capaces de seguir el orden lógico de la misma. \\
\hline
\end{tabular}

Jornadas de Formación e Innovación Docente del Profesorado I № 1 (2018)

Esta obra se distribuye con la licencia Creative Commons Reconocimiento-NoComercial-SinObraDerivada $\quad 4.0$

Internacional (CC BY-NC-ND 4.0.) 
Recursos: $\mathrm{VOZ}$

10

Fase metodológica: Ideas/hipótesis de los alumnos 40

Descripción: Les pongo un video que trata de manera muy crítica los últimos epígrafes del tema (sobre las RRII en el contexto de la globalización). El video cuestiona el modo en el que los estados occidentales se han relacionado con los estados africanos y qué efectos negativos han tenido esas relaciones en el continente africano. Tras el visionado les pido que abramos un debate para que se pronuncien sobre los temas que toca el vídeo.

Recursos: proyector, youtube https://www.youtube.com/watch?v=AfnruW7yERA

\begin{tabular}{|l|l|l|}
\hline 11 & Fase metodológica: Actividad de contraste & 20 \\
\hline
\end{tabular}

Descripción: Comentamos los puntos teóricos principales sobre el apartado de las RRII en el contexto de la globalización con la ayuda del ppt para que sea más ordenado y consistente para los alumnos. La idea es que relacionen la casuística que pone de relevancia el vídeo con los aspectos teóricos del tema.

Recursos: ppt, proyector.

12

Fase metodológica: Exposición individual noticia

$15^{\prime}$

Descripción: los dos alumnos a los que les corresponde ese día realizan su exposición breve de una noticia que hayan seleccionado previamente. Para el comentario de noticias tienen unos 5-7 minutos por persona.

Recursos: proyector, internet.

13

Fase metodológica: evaluación KAHOOT

15

Descripción: Los alumnos se autoevaluarán a través de un cuestionario breve (unas 5 preguntas tipo test) sobre la lección que 4 para acabar y cerrar la misma. La herramienta del kahoot me parece especialmente práctica para estos casos y ellos lo realizan siempre con mucho entusiasmo.

Recursos: kahoot, proyector, móviles, internet.

\section{Cuestionario previo}

Se presenta a continuación el cuestionario que se preparó para pasárselo a los alumnos el primer día del ciclo de mejora. Con este cuestionario se pretende conocer el punto de partida de los estudiantes para poder definir el enfoque de las clases y mejorar el aprendizaje (Rafael Porlán, 2017).

Jornadas de Formación e Innovación Docente del Profesorado | № 1 (2018) Esta obra se distribuye con la licencia Creative Commons Reconocimiento-NoComercial-SinObraDerivada Internacional (CC BY-NC-ND 4.0.) 
Este cuestionario no es un examen y por tanto no se tendri en cuenta para valorar la nota de la astgnatura. Responda en función de sus ideas y experiencia propia I

\title{
Identificación:
}

\section{CUESTIONARIO INICIAL SEGUNDO CICLO DE MEJORA}

El 30 de Noviembre de 2012 aparece publicada en prensa la siguiente noticia:

\begin{abstract}
"Por una abrumadora mayoria, la Asamblea General de Naciones Unidas admitió este jueves a Palestina como estado observador, lo que implicitamente supone el reconocimiento, por parte de la organización más representativa de la voluntad internacional, de la soberania de los palestinos sobre el territorio ocupado por Israel desde 1967. La votación no supone la admisión de Palestina como miembro de pleno derecho de la ONU, un paso que corresponde al Consejo de Seguridad, ni tiene consecuencias inmediatas en cuanto a la creación efectiva de un Estado. Pero da a los palestinos renovada legitimidad en su lucha contra la ocupación y llama la atención mundial sobre la urgente necesidad de poner fin a un problema que explica en gran medida el clima permanente de inestabilidad y violencia en Oriente Próximo."
\end{abstract}

En este contexto, el Presidente Palestino Mahmoud Abbas, reune a su gabinete de crisis del que usted forma parte, para redactar un discurso para la población en el que se pongan de manifiesto las principales cuestiones derivadas de la noticia y los reclamos palestinos en este sentido. Como miembro de dicho gabinete, el Presidente le pide que redacte las respuestas a las siguientes preguntas:

1. Piensa que Palestina puede constituirse como un Estado? Razone su respuesta

2. Por qué piensa que es necesario para Palestina ser considerada un Estado? Aporte todos los argumentos posibles, ordenados y sistematizados, para que puedan ser utilizados por el Presidente en su discurso

3. Piensa que el reconocimiento de la Asamblea General es relevante? Aporte todas las razones que considere puedan ser de interés para la población

4. De qué manera cree que el conflicto entre Israel y Palestina se ha visto influido por otros Estados? Incluya todas las influencias que considere relevantes y argumente cada una de ellas lo mejor posible, para que sean entendidas por la poblacion.

Figura 3. Cuestionario inicial y final

\section{Aplicación del Ciclo de Mejora}

\section{Diario de sesiones}

\section{Primera sesión}

En esta primera sesión les planteo el taller conceptual. La idea les entusiasma y veo que todos los integrantes de los grupos se implican en el trabajo asignado. La dinámica 
me resulta muy positiva porque los veo muy motivados y yo me voy acercando a cada grupo para ver cómo van avanzando. En muchos casos observo que los grupos son muy autónomos aunque en otros han necesitado más ayuda pero en general todos han conseguido llegar a los objetivos que me proponía con esta actividad. No obstante, en el debate posterior observo que al ser un tema tan candente como es el proceso independentista en Cataluña, acaban aportando en muchos casos argumentos más pasionales que teóricos sobre las cuestiones que yo les planteaba al inicio de la tarea. Tomo nota y, aunque el debate ha sido muy interesante, me planteo modificar el caso de estudio para futuros trabajos de este tipo ya que con una clase tan numerosa me resulta complicado guiar el proceso con tantas pasiones sobre la mesa. Las dos horas de clase se pasan volando tanto para ellos como para mí, de hecho alguno me manifiesta que no le ha parecido estar en clase. Entiendo que con esto se refiere al sistema tradicional, en el que el profesor transmite sin más los conceptos, por lo que el balance final de esta primera sesión es muy positivo tanto profesional como personalmente. No obstante, no da tiempo a realizar las intervenciones individuales porque, como indicaba antes, me cuesta lidiar con los tiempos cuando los veo tan implicados, así que las dejamos para la siguiente clase.

\section{Segunda sesión}

En la segunda sesión me los encuentro un poco más aletargados (es jueves a última hora) y hay bastantes faltas de asistencia. Me indican que tienen un examen al día siguiente que es el primero de la carrera y que tienen mucho miedo por lo que muchos compañeros se han quedado estudiando. Debido al retraso que acumulamos con respecto a las intervenciones individuales de la sesión anterior nos demoramos más de lo que tenía pensando en las exposiciones, pero no lo veo como un aspecto negativo porque utilizo detalles de las mismas para ir ilustrando puntos del 
temario y para aclarar otras dudas. Tras cada intervención les planteo cuestiones de actualidad e intento reconducirlas a los puntos fundamentales de la parte teórica de la lección, con lo que pueden ir conectando ideas y viendo la aplicación práctica de los contendidos teóricos. Una vez acabada esta primera parte vemos parte del temario teórico a través de la presentación ppt. En esta fase no me limito a explicar lo que previamente he incluido en las diapositivas sino que la mayoría de las mismas son imágenes (intento que esto sea así en la medida de lo posible) para de nuevo ir planteándole problemas y cuestiones que ellos puedan intentar resolver. También voy preguntando por los conceptos que vimos en la sesión anterior y me reconforta ver que muchos de ellos ya lo han asimilado. Me cuesta un poco más que se impliquen hoy, me dicen que están muy cansados y que les gusta más la dinámica del trabajo en grupo con lo que aprovecho también para hacer una mini tutoría y explicarles un poco el sentido de cada una de las clases para su aprendizaje.

\section{Tercera sesión}

En la última sesión de este ciclo de mejora comienzo preguntándoles por el examen de otra asignatura que tuvieron en la semana anterior. Con ello pretendo que vea que efectivamente me interesa su aprendizaje holístico y no solo en mi asignatura y creo que así además voy estableciendo con ellos vínculos más cercanos que pueden favorecer el aprendizaje en general. Tras dedicarle unos 5 minutos a esto, repasamos en voz alta y con voluntarios los conceptos claves vistos hasta ahora. Tras este repaso inicial, les pongo el video que les traía sobre los efectos de la globalización. Los veo muy interesados en el video, noto que este tipo de recursos audiovisuales captan su atención con más facilidad que una simple presentación PowerPoint. Tras el visionado, debatimos. Al principio les cuesta porque les da miedo decir algo inoportuno y porque en general son pocos a los que no les da miedo hablar en público. Para ello voy planteando de nuevo preguntas 
asequibles para que se vayan implicando. El resultado del debate me parece muy bueno, son capaces de pensar por ellos mismos y me parece que van desarrollando espíritu crítico. En la última media hora de clase acabamos con las exposiciones individuales, donde de nuevo utilizo los temas para repasar conceptos ya vistos no solo de esta lección sino también de las anteriores. No nos da tiempo a realizar el Kahoot, por lo que lo dejamos para la siguiente clase aunque ya no esté dentro del periodo de este ciclo de mejora, pero es un mecanismo que me está resultando muy útil para que ellos mismos se vayan evaluando. En definitiva y como conclusión general acabo muy contenta y satisfecha este segundo ciclo de mejora, por ellos y por mí misma porque voy observando las ventajas de este método y todo su potencial. No obstante veo también que solo tres sesiones son pocas para poder desarrollar una lección entera debidamente y con todas las actividades que tenía programadas. Creo que al menos 405 sesiones serían necesarias para poder realizarlo todo con más calma, así que lo apunto para la próxima.

\section{Escaleras de aprendizaje}

A partir del análisis del cuestionario previo que se pasó a los alumnos al comienzo del ciclo de mejora, pudimos establecer el punto de partida de cada uno de ellos. Para cada pregunta se establecieron las correspondientes 'escaleras de aprendizaje'. Tras la realización de todas las actividades programadas para el ciclo de mejora los alumnos volvieron a realizar el mismo cuestionario construyéndose nuevas escaleras. A continuación se representa en la figura 4:
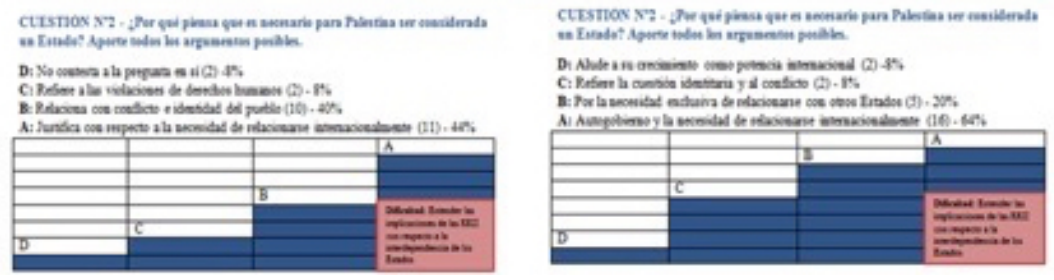

Jornadas de Formación e Innovación Docente del Profesorado I № 1 (2018)

Esta obra se distribuye con la licencia Creative Commons Reconocimiento-NoComercial-SinObraDerivada Internacional (CC BY-NC-ND 4.0.) 


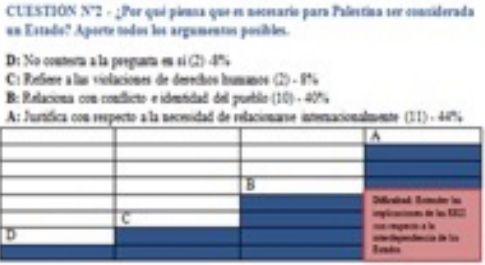

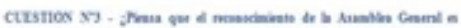

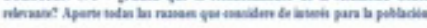

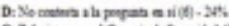

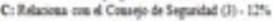

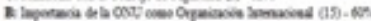

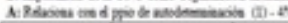

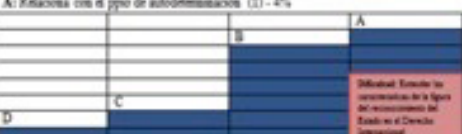

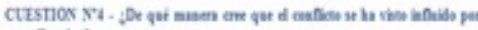
ocen furadu?

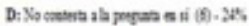

C. Ora Raven varia (f . 2as.

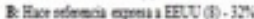

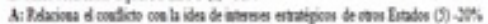

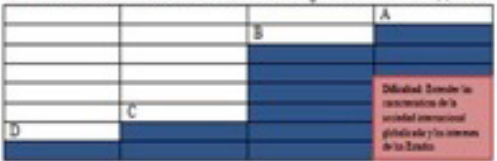

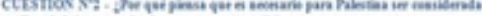

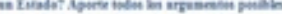

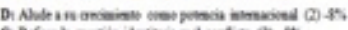

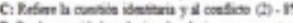

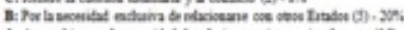

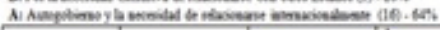

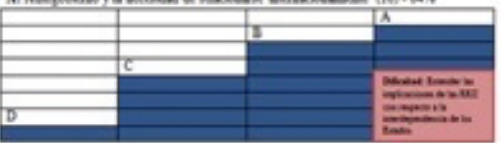

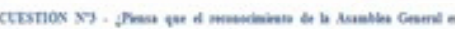

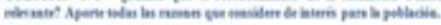

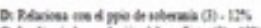

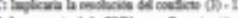

(a)

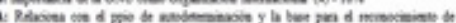

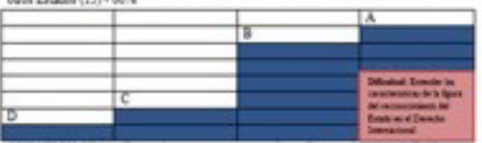

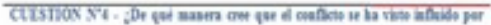
otem Entuln:

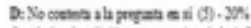

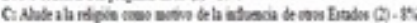

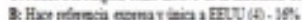

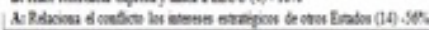

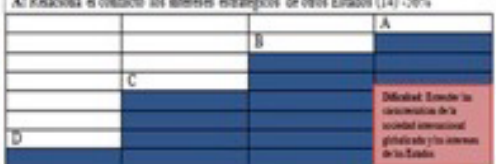

Figura 4. Escaleras de aprendizaje basadas en el análisis de los cuestionarios

La correlación de los cuestionarios iniciales y finales fue posteriormente analizada en 25 alumnos elegidos al azar, obteniéndose los resultados que se muestran en la tabla de la Figura 7.

El análisis en conjunto de las escaleras de aprendizaje revela que la gran mayoría de los alumnos han alcanzado el nivel de aprendizaje final esperado. Si bien es cierto que algunas cuestiones les han resultado más sencillas de comprender (como la figura del reconocimiento, pregunta 2), se observa que aquellas en las que se requiere un mayor desarrollo procedimental y crítico el resultado es más moderado (soberanía y globalización, por ejemplo, pregunta 4) 


\begin{tabular}{|c|c|c|c|c|c|c|}
\hline \multirow{2}{*}{$\begin{array}{l}\text { Alasas } \\
\text { I }\end{array}$} & \multicolumn{3}{|c|}{ Pregata 3} & \multicolumn{3}{|c|}{ Pegasb 4} \\
\hline & 3 & $A$ & $A$ & A & A & $\leftrightarrow$ \\
\hline 2 & 3 & A & $A$ & 3 & A & $A$ \\
\hline 3 & $c$ & A & 141 & 3 & A & $A$ \\
\hline 4 & 8 & A & $A$ & $\bar{\lambda}$ & $\bar{A}$ & $\Leftrightarrow$ \\
\hline 5 & 3 & 8 & $\longleftrightarrow$ & 3 & $\bar{A}$ & $A$ \\
\hline 6 & 3 & A & $A$ & c & $\bar{B}$ & $A$ \\
\hline 7 & $\mathrm{C}$ & $B$ & A & $C$ & B & $A$ \\
\hline 1 & D & C & A & D & C & 1 \\
\hline 9 & 3 & A & $\mathrm{A}$ & $C$ & A & 411 \\
\hline 10 & $\overline{8}$ & A & $A$ & $c$ & $\bar{A}$ & 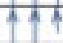 \\
\hline II & $\bar{D}$ & D & $\leftrightarrow$ & D & D & $\leftrightarrow$ \\
\hline 12 & $c$ & $B$ & $A$ & $D$ & $D$ & $\Leftrightarrow$ \\
\hline 13 & 8 & A & 4 & 8 & A & $A$ \\
\hline 14 & 3 & A & A & c & $\bar{B}$ & $A$ \\
\hline 15 & D & D & $\leftrightarrow$ & D & D & $\Leftrightarrow$ \\
\hline 16 & 8 & A & $A$ & 3 & A & $A$ \\
\hline 17 & A & A & 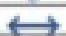 & $A$ & $A$ & $\leftrightarrow$ \\
\hline 18 & 3 & A & $A$ & $\mathrm{C}$ & A & $14 \hat{1}$ \\
\hline 19 & 8 & A & $A$ & 5 & D & $\Leftrightarrow$ \\
\hline 8 & 0 & $\mathrm{C}$ & A & D & D & $\leftrightarrow$ \\
\hline $2 \pi$ & $\bar{B}$ & A & $B$ & $\lambda$ & A & $\longleftrightarrow$ \\
\hline 21 & 0 & D & $\leftrightarrow$ & c & B & $A$ \\
\hline 2 & 0 & $\mathrm{C}$ & $A$ & D & C & $A$ \\
\hline 24 & 8 & $A$ & $A$ & A & A & $\Leftrightarrow$ \\
\hline 21 & 3 & b & $\leftrightarrow$ & 3 & A & $A$ \\
\hline
\end{tabular}

\begin{tabular}{|c|c|c|c|c|c|c|}
\hline \multirow{2}{*}{$\frac{\text { Alamo }}{1}$} & \multicolumn{3}{|c|}{ Prequata I } & \multicolumn{3}{|c|}{ Prequath 2} \\
\hline & $B$ & $\bar{A}$ & 1 & $A$ & A & $\longleftrightarrow$ \\
\hline 2 & C & A & 11 & $\bar{B}$ & $\bar{A}$ & 1 \\
\hline 3 & C & $\bar{A}$ & 11 & $\bar{B}$ & $\bar{A}$ & $i$ \\
\hline 4 & 3 & A & 1 & A & A & $\rightleftarrows$ \\
\hline 5 & $B$ & $\bar{A}$ & $A$ & $A$ & $A$ & $\longleftrightarrow$ \\
\hline 6 & C & B & 1 & B & A & 1 \\
\hline 7 & B & A & 1 & $A$ & $A$ & $\rightleftarrows$ \\
\hline 1 & C & A & 11 & B & A & $A$ \\
\hline 9 & D & D & $\longleftrightarrow$ & B & B & $\leftrightarrow$ \\
\hline 10 & 3 & A & $A$ & A & A & $\leftrightarrow$ \\
\hline II & B & A & $A$ & A & A & $\longleftrightarrow$ \\
\hline 12 & C & A & 11 & B & A & 1 \\
\hline B & C & c & $\longleftrightarrow$ & C & C & $\longleftrightarrow$ \\
\hline 11 & C & C & $\longleftrightarrow$ & D & C & 1 \\
\hline 15 & B & A & 1 & $A$ & $A$ & $\leftrightarrow$ \\
\hline 16 & C & A & 11 & 8 & 8 & $\rightleftarrows$ \\
\hline 17 & 3 & A & 1 & $A$ & $\bar{A}$ & $\longleftrightarrow$ \\
\hline 18 & C & B & 1 & B & B & $\leftrightarrow$ \\
\hline 19 & $c$ & B & 1 & $A$ & $A$ & $\leftrightarrow$ \\
\hline DD & D & A & $M M$ & B & B & $\leftrightarrow$ \\
\hline 21 & A & A & $\longleftrightarrow$ & A & A & $\longleftrightarrow$ \\
\hline 2 & C & A & 11 & C & C & $\rightleftarrows$ \\
\hline 3 & D & $B$ & 14 & D & D & $\rightleftarrows$ \\
\hline 24 & C & A & $A 14$ & $A$ & A & $\leftrightarrow$ \\
\hline 8 & C & A & An & B & B & $\longleftrightarrow$ \\
\hline
\end{tabular}

Figura 5. Análisis de la progresión de los estudiantes a través de los cuestionarios.

\section{Evaluación del diseño del ciclo de mejora}

El resultado del análisis de los cuestionarios inicial y final me parece muy prometedor, ya que la gran mayoría de los estudiantes no solo ha subido de escalón sino que un número significativo ha logrado alcanzar la respuesta más correcta. Estimo que la actividad que más ha contribuido a alcanzar los objetivos de aprendizaje fue el taller conceptual o trabajo en grupo, aunque para el curso próximo pensaré mejor la elección del tema porque el caso de Cataluña resulta tan controvertido que en ocasiones me costó guiar la clase sobre los puntos teóricos que quería desarrollar. No obstante, pienso incluir este tipo de metodología en 
todas las asignaturas que imparto porque no solo me parece útil a mí sino que observo que a los alumnos les motiva mucho trabajar de esa manera. Por otro lado, el debate que se originó en torno al video sobre los efectos de la globalización fue también muy enriquecedor. En cualquier caso, sigo encontrándome con alumnos que no terminan de encajar en este sistema de aprendizaje y que se sienten perdidos cuando la clase no se da conforme al sistema tradicional y simplemente transmisivo (Ken Bain, 2007). Convencerlos en lo que nos queda de curso de las bondades del nuevo sistema es mi reto personal.

\section{Conclusiones}

En esta comunicación se ha presentado el diseño, la aplicación y la evaluación de un ciclo de mejora docente al tema 'El Estado' de la asignatura Relaciones Internacionales del primer curso del Grado en Periodismo. Esta aproximación supone apostar por un nuevo sistema docente en el que el punto de partida son los alumnos y las cuestiones derivadas de la realidad, de la práctica, en lugar del sistema tradicional de clases magistrales cuyos resultados están quedando progresivamente en evidencia. Tanto el modelo metodológico aplicado como las actividades desarrolladas ha demostrado ser muy satisfactoria a la hora de abordar los contenidos de la lección, tal y como pone de manifiesto el análisis de los cuestionarios iniciales y finales. El mejor aval para esta nueva concepción de la docencia reside, sin duda, en los resultados de aprendizaje obtenidos y reflejados en la presente comunicación.

\section{Referencias bibliográficas.}

Don Finkel, Dar clase con la boca cerrada, Publicaciones Universidad de Valencia, 2008.

Ken Bain, Lo que hacen los mejores profesores universitarios, Publicaciones Universidad de Valencia, 2007.

Rafael Porlán (Coord.), Enseñanza Universitaria. Cómo mejorarla, Ediciones Morata, 2017. 